\title{
Exact Critical Properties of the Multi-Component Interacting Fermion Model with Boundaries
}

\author{
Satoshi Fujimoto \\ Department of Physics, Kyoto University, Kyoto 606, Japan \\ Norio Kawakami \\ Department of Applied Physics, and Department of Material and Life Science, \\ Osaka University, Suita, Osaka 565, Japan
}

\begin{abstract}
Exact critical properties of the one-dimensional $\mathrm{SU}(N)$ interacting fermion model with open boundaries are studied by using the Bethe ansatz method. We derive the surface critical exponents of various correlation functions using boundary conformal field theory. They are classified into two types, i.e. the exponents for the chiral $\mathrm{SU}(N)$ Tomonaga-Luttinger liquid and those related to the orthogonality catastrophe. We discuss a possible application of the results to the photoemission (absorption) in the edge state of the fractional quantum Hall effect.
\end{abstract}

PACS numbers: 71.27.+a, 78.66.-w

Typeset using REVTEX 


\section{INTRODUCTION}

One-dimensional (1D) quantum many-body systems with open boundaries have attracted much interest recently in connection with various problems in condensed matter physics such as the Kondo problem, the tunneling in a quantum wire and the edge state of the fractional quantum Hall effect(FQHE). A number of exactly solvable models with open boundaries have been known so far, e.g. the XXZ Heisenberg model [1], the interacting boson model [2], the interacting fermion model [3], the Hubbard model [4], and the $1 / r^{2}$ quantum model [5], etc. Quantum impurity models such as the Kondo model and the Anderson model also have a deep connection with the boundary problem [6,7]. All the above systems exhibit the surface critical behavior near the boundary. The corresponding surface exponents, which should be different from the bulk ones, can be obtained by using the finite-size scaling in boundary conformal field theory [8].

In this paper, we obtain the exact surface critical exponents for the $1 \mathrm{D} \mathrm{SU}(N)$ interacting fermion model with open boundaries by combining the Bethe ansatz solution and boundary conformal field theory. By examining boundary conditions carefully, we classify the surface exponents into two categories, i.e. the exponents for the chiral Tomonaga-Luttinger liquid and those related to the orthogonality catastrophe. The latter is shown to be related to the X-ray problem in $1 \mathrm{D}$ chiral systems. We apply our results to the edge states of the FQHE, and predict some expected behaviors for the photoemission (absorption) singularity. Some of the results obtained in this paper were previously conjectured in [7].

The plan of the paper is as follows. In sec. II, we introduce the continuum fermion model with $\mathrm{SU}(N)$ spin symmetry in open boundary conditions, and give the Bethe ansatz equations. In sec. III, based on boundary conformal field theory, we derive critical exponents for various correlation functions from the finite-size spectrum computed by the Bethe ansatz solution. In sec.IV, we discuss a possible application of the present results to the X-ray photoemission (absorption) problem in 1D chiral electron systems. A brief summary is given in sec.V. 


\section{MODEL AND BETHE ANSATZ SOLUTION}

We consider the interacting fermion model with $\mathrm{SU}(N)$ spin symmetry in open boundary conditions. The mutual electron interaction is of $\delta$-function type, and boundary potentials are introduced at both ends of the open chain. The Hamiltonian is thus given by

$H=-\sum_{m=1}^{N} \sum_{i=1}^{N_{m}} \frac{\partial^{2}}{\partial x_{i, m}^{2}}+2 c \sum_{i<j, m, n} \delta\left(x_{i, m}-x_{j, n}\right)-\sum_{m=1}^{N} \sum_{i=1}^{N_{m}}\left(\gamma_{0} \delta\left(x_{i, m}\right)+\gamma_{L} \delta\left(x_{i, m}-L\right)\right), \quad(c>0)$

where $N_{m}$ is the number of electrons with spin index $m(=1,2, \cdots, N)$ of $\operatorname{SU}(N)$ symmetry and $L$ is the system size. The last two terms represent boundary potentials with coupling constants $\gamma_{0(L)}$. We note that this type of the $\mathrm{SU}(N)$ fermion model with $\delta$-function interaction was first solved by Sutherland under the periodic boundary condition many years ago [9]. We now wish to solve the above model (1) for the open chain with boundary potentials $\gamma_{0(L)}$. This can be preformed by generalizing Gaudin's method developed for the boson model with open boundary conditions [2]. The main idea is that one can treat the open boundary problem more easily by introducing fictitious mirror-image particles with respect to the boundary. We then diagonalize the Hamiltonian (1) following standard Bethe ansatz techniques developed for periodic systems [9], and end up with the basic algebraic equations for rapidities $k_{j}$ and $\Lambda_{\alpha}^{(l)}$,

$$
\begin{aligned}
& 2 L k_{j}+\varphi_{0}\left(k_{j}\right)+\varphi_{L}\left(k_{j}\right)=2 \pi I_{j}-\sum_{\beta=1}^{M_{1}}\left(2 \tan ^{-1}\left[\frac{2\left(k_{j}-\Lambda_{\beta}^{(1)}\right)}{c}\right]+2 \tan ^{-1}\left[\frac{2\left(k_{j}+\Lambda_{\beta}^{(1)}\right)}{c}\right]\right) \\
& \sum_{\beta=1}^{M_{l-1}}\left(2 \tan ^{-1}\left[\frac{2\left(\Lambda_{\alpha}^{(l)}-\Lambda_{\beta}^{(l-1)}\right)}{c}\right]+2 \tan ^{-1}\left[\frac{2\left(\Lambda_{\alpha}^{(l)}+\Lambda_{\beta}^{(l-1)}\right)}{c}\right]\right)=2 \pi J_{\alpha}^{(l)} \\
& +\sum_{\beta=1, \beta \neq \alpha}^{M_{l}}\left(2 \tan ^{-1}\left[\frac{\Lambda_{\alpha}^{(l)}-\Lambda_{\beta}^{(l)}}{c}\right]+2 \tan ^{-1}\left[\frac{\Lambda_{\alpha}^{(l)}+\Lambda_{\beta}^{(l)}}{c}\right]\right) \\
& -\sum_{\beta=1}^{M_{l+1}}\left(2 \tan ^{-1}\left[\frac{2\left(\Lambda_{\alpha}^{(l)}-\Lambda_{\beta}^{(l+1)}\right)}{c}\right]+2 \tan ^{-1}\left[\frac{2\left(\Lambda_{\alpha}^{(l)}+\Lambda_{\beta}^{(l+1)}\right)}{c}\right]\right), \\
& 1 \leq l \leq N-1, \quad \alpha=1,2, \ldots, M_{l},
\end{aligned}
$$

with $M_{N} \equiv 0, M_{l}=\sum_{\alpha=l+1}^{N} N_{\alpha}(0 \leq l \leq N-1)$ and $\Lambda_{j}^{(0)} \equiv k_{j}$, where $\varphi_{p}(k)=-2 \tan ^{-1} \frac{k}{\gamma_{p}}$, $(p=0, L)$ are the phase shifts due to the boundary scattering. Quantum numbers $I_{j}$ and 
$J_{\alpha}^{(l)}$ are positive integers (or half-odd integers) which classify the elementary excitations. The total energy is simply given by

$$
E=\sum_{j=1}^{N} k_{j}^{2} .
$$

Note that the above equations contain the terms with arguments like $\Lambda_{\alpha}^{l}+\Lambda_{\beta}^{l}$, reflecting the introduction of image particles [2 [4], in contrast to the ordinary Bethe ansatz equations for the periodic case. These equations for the $\mathrm{SU}(2)$ case were obtained firstly by Woynarovich [3]. Defining new variables, $\Lambda_{-\alpha}^{(l)}=-\Lambda_{\alpha}^{(l)}$, we can rewrite the above equations into more tractable form,

$$
\begin{aligned}
2 L k_{j}+\varphi_{0}\left(k_{j}\right)+\varphi_{L}\left(k_{j}\right)=2 \pi I_{j} & +2 \tan ^{-1}\left(\frac{2 k_{j}}{c}\right)-\sum_{\beta=-M_{1}}^{M_{1}} 2 \tan ^{-1}\left[\frac{2\left(k_{j}-\Lambda_{\beta}^{(1)}\right)}{c}\right], \\
\sum_{\beta=-M_{l-1}}^{M_{l-1}} 2 \tan ^{-1}\left[\frac{2\left(\Lambda_{\alpha}^{(l)}-\Lambda_{\beta}^{(l-1)}\right)}{c}\right] & =2 \pi J_{\alpha}-2 \tan ^{-1}\left(\frac{\Lambda_{\alpha}^{(l)}}{c}\right)+2 \tan ^{-1}\left(\frac{2 \Lambda_{\alpha}^{(l)}}{c}\right) \\
+\sum_{\beta=-M_{l}}^{M_{l}} 2 \tan ^{-1}\left[\frac{\Lambda_{\alpha}^{(l)}-\Lambda_{\beta}^{(l)}}{c}\right] & -\sum_{\beta=-M_{l+1}}^{M_{l+1}} 2 \tan ^{-1}\left[\frac{2\left(\Lambda_{\alpha}^{(l)}-\Lambda_{\beta}^{(l+1)}\right)}{c}\right], \quad 1 \leq l \leq N-2, \\
\sum_{\beta=-M_{N-2}}^{M_{N-2}} 2 \tan ^{-1}\left[\frac{2\left(\Lambda_{\alpha}^{(N-1)}-\Lambda_{\beta}^{(N-2)}\right)}{c}\right] & =2 \pi J_{\alpha}-2 \tan ^{-1}\left(\frac{\Lambda_{\alpha}^{(N-1)}}{c}\right) \\
& +\sum_{\beta=-M_{N-1}}^{M_{N-1}} 2 \tan ^{-1}\left[\frac{\Lambda_{\alpha}^{(N-1)}-\Lambda_{\beta}^{(N-1)}}{c}\right] .
\end{aligned}
$$

After this transformation, the structure of the equations formally resembles to that for the periodic case [9]. This fact makes the following analysis much easier .

In the following, we will be concerned with the case of repulsive boundary potentials $\left(\gamma_{p}<0\right)$ for simplicity. All the rapidities then turn out to be real in this case. Taking the thermodynamic limit, we now recast the algebraic equations (5) (7) into the integral equations for the density functions of rapidities,

$$
\begin{aligned}
2 \pi \rho(k)= & 2+\frac{1}{L}\left(\varphi_{0}^{\prime}(k)+\varphi_{L}^{\prime}(k)\right)-\frac{1}{L} \frac{c}{(c / 2)^{2}+k^{2}} \\
& +\int_{\Lambda_{-}^{(1)}}^{\Lambda_{+}^{(1)}} d \Lambda \frac{c}{(c / 2)^{2}+(k-\Lambda)^{2}} \sigma^{(1)}(\Lambda), \\
2 \pi \sigma^{(l)}\left(\Lambda^{(l)}\right)= & \frac{1}{L} \frac{2 c}{c^{2}+\left(\Lambda^{(l)}\right)^{2}}-\frac{1}{L} \frac{c}{(c / 2)^{2}+\left(\Lambda^{(l)}\right)^{2}}-\int_{\Lambda_{-}^{(l)}}^{\Lambda_{+}^{(l)}} d \Lambda^{\prime} \frac{2 c}{c^{2}+\left(\Lambda^{(l)}-\Lambda^{\prime}\right)^{2}} \sigma^{(l)}\left(\Lambda^{\prime}\right)
\end{aligned}
$$




$$
\begin{aligned}
& +\int_{\Lambda_{-}^{(l-1)}}^{\Lambda_{+}^{(l-1)}} d \Lambda^{\prime} \frac{c}{(c / 2)^{2}+\left(\Lambda^{(l)}-\Lambda^{\prime}\right)^{2}} \sigma^{(l-1)}\left(\Lambda^{\prime}\right) \\
& +\int_{\Lambda_{-}^{(l+1)}}^{\Lambda_{+}^{(l+1)}} d \Lambda^{\prime} \frac{c}{(c / 2)^{2}+\left(\Lambda^{(l)}-\Lambda^{\prime}\right)^{2}} \sigma^{(l+1)}\left(\Lambda^{\prime}\right), \quad 1 \leq l \leq N-2 \\
& 2 \pi \sigma^{(N-1)}\left(\Lambda^{(N-1)}\right)=\frac{1}{L} \frac{2 c}{c^{2}+\left(\Lambda^{(l)}\right)^{2}}-\int_{\Lambda_{-}^{(N-1)}}^{\Lambda_{+}^{(N-1)}} d \Lambda^{\prime} \frac{2 c}{c^{2}+\left(\Lambda^{(N-1)}-\Lambda^{\prime}\right)^{2}} \sigma^{(N-1)}\left(\Lambda^{\prime}\right) \\
& +\int_{\Lambda_{-}^{(N-2)}}^{\Lambda_{+}^{(N-2)}} d \Lambda^{\prime} \frac{c}{(c / 2)^{2}+\left(\Lambda^{(N-2)}-\Lambda^{\prime}\right)^{2}} \sigma^{(N-2)}\left(\Lambda^{\prime}\right)
\end{aligned}
$$

where $\Lambda_{+}^{(0)}=-\Lambda_{-}^{(0)}=k_{F}^{(c)}$, which is determined by the condition,

$$
\int_{-k_{F}^{(c)}}^{k_{F}^{(c)}} d k \rho(k)=\frac{2 M_{0}+1}{L} .
$$

In the absence of magnetic fields, one can see that $\Lambda_{+}^{(l)}=-\Lambda_{-}^{(l)} \rightarrow+\infty(1 \leq l \leq N-1)$, and the system recovers $\mathrm{SU}(N)$ spin symmetry. It is convenient to divide the density functions into bulk and boundary parts,

$$
\rho(k) \equiv \rho_{b u l k}(k)+\frac{1}{L} \rho_{b}(k)
$$

In zero magnetic field, the coupled integral equations for the density functions can be reduced to simple ones for $\rho_{b u l k}$ and $\rho_{b}$ from eqs.(\$) $\sim(10)$ by using Fourier transformation. The results are

$$
\begin{gathered}
2 \pi \rho_{\text {bulk }}(k)=2+\int_{-k_{F}^{(c)}}^{k_{F}^{(c)}} d k^{\prime} G\left(k-k^{\prime}\right) \rho_{\text {bulk }}\left(k^{\prime}\right), \\
2 \pi \rho_{b}(k)=\varphi_{0}^{\prime}(k)+\varphi_{L}^{\prime}(k)-\frac{c}{(c / 2)^{2}+k^{2}}+\int_{-\infty}^{\infty} d x e^{-i k x} f(x)+\int_{-k_{F}^{(c)}}^{k_{F}^{(c)}} d k^{\prime} G\left(k-k^{\prime}\right) \rho_{b}\left(k^{\prime}\right),
\end{gathered}
$$

where the integral kernel is

$$
\begin{gathered}
G(k)=\int_{-\infty}^{\infty} d x e^{i k x} e^{-\frac{c}{2}|x|} \frac{\sinh ((N-1) c x / 2)}{\sinh (N c x / 2)}, \\
f(x)=\frac{e^{-c|x|}-e^{-\frac{c}{2}|x|}}{2 \sinh \frac{c x}{2} \sinh \frac{N c x}{2}}\left(\cosh \frac{N c x}{2}+\cosh \frac{(N-1) c x}{2}-\cosh c x-\cosh \frac{c x}{2}\right)+e^{-c|x|} \frac{\sinh \frac{c x}{2}}{\sinh \frac{N c x}{2}} .
\end{gathered}
$$


The total energy is given by

$$
\frac{E}{L}=\int_{-k_{F}^{(c)}}^{k_{F}^{(c)}} d k \rho(k) k^{2} .
$$

Eqs.(13) (17) determine the energy spectrum of the model. It should be noticed that in the thermodynamic limit, the bulk properties of the present model is the same as those for the periodic model [9], which have been already studied in detail [10]. To examine critical properties, we thus need to study the finite-size spectrum, which will be done below.

\section{BOUNDARY CRITICAL PROPERTIES}

In this section, we compute the energy spectrum for the finite system, and discuss lowenergy critical properties using boundary conformal field theory. We then obtain the surface critical exponents of various correlation functions.

\section{A. finite-size spectrum and conformal properties}

Applying a standard technique in the Bethe ansatz method [11], we now obtain the finite-size energy spectrum from the basic equations derived in the previous section. For this purpose, let us first express the total energy, eq.(17), in terms of the dressed energies,

$$
\begin{aligned}
\frac{E}{L}= & \int_{-k_{F}^{(c)}}^{k_{F}^{(c)}} d k\left[\frac{1}{\pi}+\frac{1}{2 \pi L}\left(\varphi_{0}^{\prime}(k)+\varphi_{L}^{\prime}(k)\right)-\frac{1}{2 \pi L} \frac{c}{(c / 2)^{2}+k^{2}}\right] \varepsilon^{(0)}(k) \\
& +\sum_{l=1}^{N-2} \int_{\Lambda_{-}^{(l)}}^{\Lambda_{+}^{(l)}} d \Lambda\left[\frac{1}{2 \pi L} \frac{2 c}{c^{2}+\Lambda^{2}}-\frac{1}{2 \pi L} \frac{c}{(c / 2)^{2}+\Lambda^{2}}\right] \varepsilon^{(l)}(\Lambda) \\
& +\int_{\Lambda_{-}^{(N-1)}}^{\Lambda_{+}^{(N-1)}} d \Lambda \frac{1}{2 \pi L} \frac{2 c}{c^{2}+\Lambda^{2}} \varepsilon^{(N-1)}(\Lambda),
\end{aligned}
$$

where the dressed energies $\varepsilon^{(l)}$ are determined by the following integral equations,

$$
\begin{aligned}
& \varepsilon^{(0)}(k)=k^{2}+\int_{\Lambda_{-}^{(1)}}^{\Lambda_{+}^{(1)}} \frac{d \Lambda}{2 \pi} \frac{c}{(c / 2)^{2}+(k-\Lambda)^{2}} \varepsilon^{(1)}(\Lambda) \\
& \varepsilon^{(l)}(\Lambda)=-\int_{\Lambda_{-}^{(l)}}^{\Lambda_{+}^{(l)}} \frac{d \Lambda^{\prime}}{2 \pi} \frac{2 c}{c^{2}+\left(\Lambda^{(l)}-\Lambda^{\prime}\right)^{2}} \varepsilon^{(l)}\left(\Lambda^{\prime}\right)
\end{aligned}
$$




$$
\begin{aligned}
& +\int_{\Lambda_{-}^{(l-1)}}^{\Lambda_{+}^{(l-1)}} \frac{d \Lambda^{\prime}}{2 \pi} \frac{c}{(c / 2)^{2}+\left(\Lambda^{(l)}-\Lambda^{\prime}\right)^{2}} \varepsilon^{(l-1)}\left(\Lambda^{\prime}\right) \\
& +\int_{\Lambda_{-}^{(l+1)}}^{\Lambda^{(l+1)}} \frac{d \Lambda^{\prime}}{2 \pi} \frac{c}{(c / 2)^{2}+\left(\Lambda^{(l)}-\Lambda^{\prime}\right)^{2}} \varepsilon^{(l+1)}\left(\Lambda^{\prime}\right), \quad 1 \leq l \leq N-2 \\
\varepsilon^{(N-1)}(\Lambda) & =-\int_{\Lambda_{-}^{(N-1)}}^{\Lambda_{+}^{(N-1)}} d \Lambda^{\prime} \frac{2 c}{c^{2}+\left(\Lambda^{(N-1)}-\Lambda^{\prime}\right)^{2}} \varepsilon^{(N-1)}\left(\Lambda^{\prime}\right) \\
& +\int_{\Lambda_{-}^{(N-2)}}^{\Lambda_{+}^{(N-2)}} d \Lambda^{\prime} \frac{c}{(c / 2)^{2}+\left(\Lambda^{(N-2)}-\Lambda^{\prime}\right)^{2}} \varepsilon^{(N-2)}\left(\Lambda^{\prime}\right) .
\end{aligned}
$$

The equivalence of the two expressions (17) and (18) can be easily checked by directly comparing them after formally solving the integral equations by the iteration scheme. The formula (18) is particularly useful to compute the excitation spectrum.

Let us start with the corrections to the ground state energy. By directly applying the Euler-Maclaurin formula,

$$
\frac{1}{N} \sum_{n=a}^{b} f\left(\frac{n}{N}\right) \sim \int_{(a-1 / 2) / N}^{(b+1 / 2) / N} f(x) d x+\frac{1}{24 N^{2}}\left(f^{\prime}\left(\frac{a-1 / 2}{N}\right)-f^{\prime}\left(\frac{b+1 / 2}{N}\right)\right),
$$

to eq.(4), we easily find the finite-size corrections to the ground state energy [12],

$$
\Delta E_{g}=-\sum_{l=0}^{N-1} \frac{\pi v_{l}}{24 L}
$$

which is scaled by the velocities $v_{l}(l=0,1, \cdots, N-1)$ defined by

$$
v_{l}=\frac{1}{2 \pi \sigma^{(l)}\left(\Lambda_{+}^{(l)}\right)} \frac{\partial \varepsilon^{(l)}\left(\Lambda_{+}^{(l)}\right)}{\partial \Lambda^{(l)}},
$$

with $\sigma^{(0)}\left(\Lambda^{(0)}\right) \equiv \rho(k)$. By exploiting the finite-size scaling for open boundaries [13, 8], we can see from eq.(23) that the Virasoro central charge for the charge sector $(l=0)$ is given by $c=1$. On the other hand, all the velocities of the spin excitation takes the same value $(l=1,2, \cdots, N-1)$ in the absence of magnetic fields, and thus the central charge for the spin sector turns out to be $c=N-1$, namely, the rank of $\mathrm{SU}(N)$ Lie algebra. We shall study conformal properties in more detail by examining the excitation spectrum below.

Elementary excitations are classified into two types, i.e. those for primary fields and for descendant fields. The former can be described by the excitations which change the number of particles. They are computed by changing the cut-off parameters in (18) as $\Lambda_{ \pm}^{(l)} \rightarrow$ 
$\Lambda_{ \pm}^{(l)}+\Delta \Lambda_{ \pm}^{(l)}$. On the other hand, the excitations for descendant fields are simply given by the particle-hole type excitations with the fixed number of particles. These manipulations are performed straightforwardly, and we end up with the finite-size spectrum for the excitation energy,

$$
\frac{\Delta E}{L}=\frac{\pi}{L}\left[\frac{1}{2} \Delta \mathbf{M}^{T}\left(\hat{\xi}^{-1}\right)^{T} V\left(\hat{\xi}^{-1}\right) \Delta \mathbf{M}+\sum_{l=0}^{N-1} v_{l} n_{+}^{(l)}\right]
$$

with $V=\operatorname{diag}\left(v_{0}, v_{1}, \ldots, v_{N-1}\right)$, where $n_{+}^{(l)}$ are non-negative integers denoting particle-hole excitations. Here $\hat{\xi}$ is the $N \times N$ matrix of the so-called dressed charge [14], whose components $\xi_{i j} \equiv \xi_{i j}\left(\Lambda_{+}^{(i)}\right)(0 \leq i, j \leq N-1)$ are determined by the following integral equations,

$$
\begin{aligned}
\xi_{0 j}(k) & =\delta_{0 j}+\int_{\Lambda_{-}^{(1)}}^{\Lambda_{+}^{(1)}} \frac{d \Lambda}{2 \pi} \frac{c}{(c / 2)^{2}+(k-\Lambda)^{2}} \xi_{1 j}(\Lambda) \\
\xi_{i j}\left(\Lambda^{(i)}\right) & =\delta_{i j}-\int_{\Lambda_{-}^{(i)}}^{\Lambda_{+}^{(i)}} \frac{d \Lambda^{\prime}}{2 \pi} \frac{2 c}{c^{2}+\left(\Lambda^{(l)}-\Lambda^{\prime}\right)^{2}} \xi_{i j}\left(\Lambda^{\prime}\right) \\
& +\int_{\Lambda_{-}^{(i-1)}}^{\Lambda_{+}^{(i-1)}} \frac{d \Lambda^{\prime}}{2 \pi} \frac{c}{(c / 2)^{2}+\left(\Lambda^{(i)}-\Lambda^{\prime}\right)^{2}} \xi_{i-1 j}\left(\Lambda^{\prime}\right) \\
& +\int_{\Lambda_{-}^{(i+1)}}^{\Lambda_{+}^{(i+1)}} \frac{d \Lambda^{\prime}}{2 \pi} \frac{c}{(c / 2)^{2}+\left(\Lambda^{(i)}-\Lambda^{\prime}\right)^{2}} \xi_{i+1 j}\left(\Lambda^{\prime}\right), \quad 1 \leq i \leq N-2 \\
\xi_{N-1 j}\left(\Lambda^{(N-1)}\right) & =\delta_{N-1 j}-\int_{\Lambda_{-}^{(N-1)}}^{\Lambda_{+}^{(N-1)}} d \Lambda^{\prime} \frac{2 c}{c^{2}+\left(\Lambda^{(N-1)}-\Lambda^{\prime}\right)^{2}} \xi_{N-1 j}\left(\Lambda^{\prime}\right) \\
& +\int_{\Lambda_{-}^{(N-2)}}^{\Lambda_{+}^{(N-2)}} d \Lambda^{\prime} \frac{c}{(c / 2)^{2}+\left(\Lambda^{(N-2)}-\Lambda^{\prime}\right)^{2}} \xi_{N-2 j}\left(\Lambda^{\prime}\right) .
\end{aligned}
$$

In eq.(25), the quantum numbers classifying elementary excitations are defined as

$$
\begin{aligned}
& \Delta M^{(l)} \equiv \Delta M_{h}^{(l)}-\frac{n_{b}}{N}(N-l), \quad 1 \leq l \leq N-1, \\
& \Delta M^{(0)}=\Delta N_{h}-n_{b} .
\end{aligned}
$$

Here $n_{b}$ is the number of particles localized at boundaries, which is given by

$$
n_{b}=\frac{1}{2} \int_{-k_{F}^{(c)}}^{k_{F}^{(c)}} d k \rho_{b}(k)
$$

and $\Delta N_{h}$ is an integer specifying charge excitations, whereas $\Delta M_{h}^{(l)}$ 's are integers which label $(N-1)$ kinds of spin excitations. 
We can now read off conformal weights $\Delta_{b}$ from eq.(25), using finite-size scaling arguments [15]. Surface critical properties near the boundary are determined by boundary scaling operators $\phi(t)$. The critical exponent for correlation functions of a boundary operator, $\langle\phi(t) \phi(0)\rangle \sim 1 / t^{x}$, is given by

$$
x=2 \Delta_{b}=\Delta \mathbf{M}^{T} \mathcal{C}_{f} \Delta \mathbf{M}+2 \sum_{l=0}^{N-1} n_{+}^{(l)},
$$

where the $N \times N$ matrix $\mathcal{C}_{f}=\left(\hat{\xi}^{-1}\right)^{T} \hat{\xi}^{-1}$ is given in the absence of magnetic fields,

$$
\mathcal{C}_{f}=\left(\begin{array}{cccc}
\frac{1}{N K_{\rho}}+\frac{N-1}{N} & -1 & & 0 \\
-1 & 2 & \ddots & \\
& \ddots & \ddots & -1 \\
0 & & -1 & 2
\end{array}\right)
$$

Here $K_{\rho} \equiv \xi_{00}^{2}\left(k_{F}^{(c)}\right) / N$ is the dimensionless coupling constant for the charge sector (socalled Tomonaga-Luttinger parameter), where the dressed charge $\xi_{00}(k)$ is determined by the integral equation,

$$
\xi_{00}(k)=1+\int_{-k_{F}^{(c)}}^{k_{F}^{(c)}} d k G\left(k-k^{\prime}\right) \xi_{00}\left(k^{\prime}\right) .
$$

Note that the system is now regarded to be chiral due to the presence of open boundaries, and quantum numbers carrying currents do not appear in the conformal weights (31). This implies that an effective theory of the present model is given by the holomorphic piece of conformal field theory. From eqs.(31) and (32), we can see that critical properties of the charge and spin sectors are respectively described by the U(1) Gaussian model with the central charge $c=1$ and the level-1 SU(N) Wess-Zumino-Witten model with $c=N-1$.

The expression (31) with (32) is one of the main results in this paper. We wish to emphasize that this formula is quite general and is applicable to many other $\mathrm{SU}(N)$ quantum models with boundaries such as the $t$ - $J$ model, the Hubbard model [⿴囗十, etc. 


\section{B. surface critical exponents}

We are now ready to obtain the surface critical exponents of various correlation functions. As is seen from eq.(29), the effects of boundary potentials are just to shift the quantum number as $\Delta M^{(l)} \rightarrow \Delta M^{(l)}-\frac{n_{b}}{N}(N-l)$. One readily notices that such an effect of boundary potentials is equivalent to that of twisting boundary conditions, which does not change the critical exponents in general. Thus when we determine the critical exponents from (31), we should discard $n_{b}$-dependence in $\Delta \mathbf{M}$ by redefining the quantum number as $\Delta M^{(l)}-$ $\frac{n_{b}}{N}(N-l) \rightarrow \Delta M^{(l)}$. Therefore, for the long-time behavior of the single-particle Green function, $\left\langle c_{\alpha}^{\dagger}(t) c_{\alpha}(0)\right\rangle \sim 1 / t^{\eta}$, we obtain its critical exponent by setting $\Delta N_{h}=1, \Delta M_{h}^{(l)}=0$ $(1 \leq l \leq N-1)$

$$
\eta=\frac{1}{N K_{\rho}}+\frac{N-1}{N} .
$$

Furthermore it is seen that the density-density correlation function and the spin-spin correlation function show the following asymptotic behavior, by taking $\Delta N_{h}=0, \Delta M_{h}^{(l)}=0$ $(1 \leq l \leq N-1)$, and $n_{+}^{(l)}=1$,

$$
\langle O(t) O(0)\rangle \sim \text { constant }+\frac{1}{t^{2}}
$$

Namely, this asymptotic behavior is determined by descendants of the primary field with $\Delta_{b}=0$, and hence the critical exponent takes the canonical (integer) values. The anomalous exponent appears only in the single-particle Green function. These characteristic properties are inherent in the chiral Tomonaga-Luttinger liquid [21], which is quite contrasted to ordinary periodic systems as will be seen in the next section.

We wish to stress that the formula (31) for conformal dimensions possesses another important information for boundary critical properties related to the orthogonality catastrophe. This is realized when one considers the problem in which the boundary potentials are time-dependent [22,7]. For example, suppose that the boundary potentials are suddenly turned on at $t_{0}$. Then the long-time asymptotic behavior of correlation functions 
$\left\langle O\left(t_{2}\right) O\left(t_{1}\right)\right\rangle$ for $t_{1} \ll t_{0} \ll t_{2}$ should show quite different properties from (34) and (35). In this case, the phase shift caused by boundary potentials plays an essential role, and then $n_{b}$ cannot be ignored by redefining the quantum numbers. Therefore, retaining $n_{b}$ in (31), we get anomalous exponents for various correlation functions. We show several examples below.

(i) single-particle Green function: $\left\langle c_{\alpha}^{\dagger}(t) c_{\alpha}(0)\right\rangle \sim 1 / t^{\eta}$. Putting $\Delta N_{h}=1, \Delta M_{h}^{(l)}=0$ $(1 \leq l \leq N-1)$, we have

$$
\eta=\frac{1}{N K_{\rho}}\left(1-n_{b}\right)^{2}+\frac{N-1}{N}
$$

(ii) density-density correlation function: $\langle n(t) n(0)\rangle \sim 1 / t^{\alpha_{c}},\left(n=\sum_{\alpha} c_{\alpha}^{\dagger} c_{\alpha}\right)$. Taking $\Delta N_{h}=$ $0, \Delta M_{h}^{(l)}=0(1 \leq l \leq N-1)$, we then obtain

$$
\alpha_{c}=\frac{n_{b}^{2}}{N K_{\rho}}
$$

(iii) spin-spin correlation function: $\left\langle S^{a}(t) S^{a}(0)\right\rangle \sim 1 / t^{\alpha_{s}},\left(S^{a}=\sum_{\alpha, \beta} c_{\alpha}^{\dagger} \tau_{\alpha \beta}^{a} c_{\beta}\right.$ with $\tau_{\alpha \beta}^{a}$ being a fundamental representation of $\mathrm{SU}(N))$. Putting $\Delta N_{h}=0, \Delta M_{h}^{(l)}=0(1 \leq l \leq N-1)$, we have

$$
\alpha_{s}=\frac{n_{b}^{2}}{N K_{\rho}} .
$$

Thus the surface critical exponents depend not only on the Tomonaga-Luttinger parameter $K_{\rho}$ but also on the fractional number $n_{b}$ of localized particles at the boundary. We will see in the next section that these critical properties have a deep connection to the X-ray edge problem in 1D chiral systems.

We can evaluate the above exponents easily in some limiting cases. In the case of noninteracting electrons $(c=0)$, we see $K_{\rho}=1$ from (33), and the critical exponents (34), (35) (36), (37) and (38) are all reduced to those obtained previously for the single-impurity $\mathrm{SU}(N)$ Anderson model with infinitely strong Coulomb interaction [7]. On the other hand, in the strong-coupling limit $c \rightarrow+\infty$ or low-density limit $k_{F}^{(c)} \rightarrow 0$, it is easily seen from eq.(33) that $K_{\rho}=1 / N$, and so the exponent of the single-particle Green function eq.(34) 
is $\eta=2-1 / N$. In general, $K_{\rho}$ may change in the range $[1,2-1 / N]$ according to the interaction strength and the density of particles.

\section{C. comparison with the periodic model}

To conclude this section, we compare the above results with bulk critical exponents under periodic boundary conditions. The finite-size spectrum for the $\mathrm{SU}(N)$ model in the periodic boundary conditions is given by [14]

$$
\frac{E}{L}=\frac{2 \pi}{L}\left(\frac{1}{4} \Delta \mathbf{M}^{T}\left(\hat{\xi}^{-1}\right)^{T} V\left(\hat{\xi}^{-1}\right) \Delta \mathbf{M}+\mathbf{D}^{T} \hat{\xi} V \hat{\xi}^{T} \mathbf{D}+\sum_{l=0}^{N-1} v_{l}\left(n_{+}^{(l)}+n_{-}^{(l)}\right)\right),
$$

where the newly introduced quantum numbers, $\mathbf{D}^{T}=\left(D_{0}, D_{1}, \ldots, D_{N-1}\right)$, are given by

$$
\begin{gathered}
D_{0}=\frac{\Delta M^{(0)}+\Delta M^{(1)}}{2}, \quad(\bmod 1), \\
D_{l}=\frac{\Delta M^{(l-1)}+\Delta M^{(l+1)}}{2}, \quad(\bmod 1), \quad l=1, \ldots, N-1,
\end{gathered}
$$

with $\Delta M^{(N)} \equiv 0$. These quantum numbers carry the large momentum transfer [14].

Using finite-size scaling arguments for periodic systems [15], we have the conformal dimensions of scaling operators,

$$
\Delta_{+}+\Delta_{-}=\frac{1}{4} \Delta \mathbf{M}^{T} \mathcal{C}_{f} \Delta \mathbf{M}+\mathbf{D}^{T} \mathcal{C}_{f}^{-1} \mathbf{D}+\sum_{l=0}^{N-1}\left(n_{+}^{(l)}+n_{-}^{(l)}\right) .
$$

From this formula, we obtain critical exponents. We list several examples in the following.

(i) The single-particle Green function:

$$
\eta=\frac{1}{2 N}\left(\frac{1}{K_{\rho}}+K_{\rho}\right)+\frac{N-1}{N} .
$$

In the strong-coupling limit or low-density limit, this reduces to [16, 17]

$$
\eta=\frac{3}{2}-\frac{1}{N}+\frac{1}{2 N^{2}} .
$$

(ii) The $2 N k_{F}$-oscillating term $\left(k_{F}\right.$ : Fermi momentum) of the density-density correlation function: 


$$
\alpha_{c}=2 N K_{\rho} .
$$

We have $\alpha_{c}=2$ in the strong-coupling limit or low-density limit.

(iii) The $2 k_{F}-$ oscillating term of the spin-spin correlation function:

$$
\alpha_{s}=2 \frac{K_{\rho}+N-1}{N},
$$

which reduces to

$$
\alpha_{s}=2\left(1-\frac{1}{N}+\frac{1}{N^{2}}\right)
$$

in the strong-coupling limit or low-density limit [16]

Comparing these results with the previous ones, we can see that the bulk critical exponents are quite different from the boundary ones, though bulk thermodynamic properties should exhibit the same behavior in both cases.

\section{APPLICATION TO THE EDGE STATE OF FQHE}

In this section, we briefly discuss a possible application of the results obtained in the previous section to the edge state of the FQHE as a typical example of 1D chiral fermion systems [21]. We first note that if an appropriate value is chosen for the Tomonaga-Luttinger parameter $K_{\rho}$, the above $\mathrm{SU}(N)$ model with open boundaries can give an effective theory for the edge state of a certain hierarchy in the FQHE. Namely, the edge state of the fractional quantum Hall effect with filling $\nu=N /(N m+1)$ ( $m$ even) can be modeled by the above open system by choosing $K_{\rho}$ as $K_{\rho}=\nu / N$ [7]. In fact, one can easily check that by this

choice of $K_{\rho}$, the formulas (34) and (35) exactly reproduce the critical exponents for the chiral liquids proposed for the edge states [21].

We shall focus on the X-ray photoemission (absorption) problem in edge states. The Fermi-edge singularity problem in 1D electron systems has attracted current interest [18 20]. In the edge state of the FQHE, electrons move only in one direction and the backward scattering due to impurities is irrelevant, and hence the system can be treated as a chiral system. 
In experiments of the X-ray photoemission or absorption, the core hole may be suddenly created, resulting in a problem with time-dependent boundary conditions [7,22]. That is, for $t<t_{0}$ the boundary is free, and at $t=t_{0}$ the boundary potential suddenly switches on. Then bulk electrons show critical low-energy behavior inherent in the orthogonality catastrophe.

In the X-ray absorption problem, one electron is excited in the final state. Thus putting $\Delta N_{h}=1, \Delta M_{h}^{(l)}=0(1 \leq l \leq N-1)$, we have the critical exponent for the X-ray absorption in this system,

$$
\alpha_{a b}=\frac{1}{\nu}\left(1-\frac{N \delta}{\pi}\right)^{2}+\frac{N-1}{N}
$$

for the filling $\nu=N /(N m+1)$ with even $m$, where $\delta$ is the phase shift caused by the localized electrons which screen the core hole potential. On the other hand, the critical exponent for the photoemission is obtained by taking $\Delta N_{h}=0$ and $\Delta M_{h}^{(l)}=0$ in eq.(31), because one hole carrying neither charge nor spin is generated in the final state,

$$
\alpha_{p h}=\frac{N^{2}}{\nu}\left(\frac{\delta}{\pi}\right)^{2}
$$

We expect that such anomalous exponents may be observed in the X-ray photoemission or absorption experiments for the edge state of the FQHE of the filling $\nu=N /(N m+1)$ with even $m$.

\section{SUMMARY}

We have studied exact boundary critical properties of the $\mathrm{SU}(N)$ interacting fermion model with open boundaries by using the Bethe ansatz solution and boundary conformal field theory. It has been shown that the surface exponents which govern the critical behavior near the boundary depend on the two continuously varying quantities, i.e. the dimensionless Tomonaga-Luttinger parameter $K_{\rho}$ and the fractional number of localized electrons at the boundary. We have also discussed a possible application of the results to the Fermi-edge singularity problem in the edge state of the FQHE as a typical example of 1D chiral systems. The exact exponents for the X-ray absorption and photoemission have been derived. 


\section{ACKNOWLEDGMENTS}

We wish to express our sincere thanks to T. Fukui, T. Yamamoto and S.-K. Yang for

valuable discussions. This work was partly supported by a Grant-in-Aid from the Ministry of Education, Science and Culture. 


\section{REFERENCES}

[1] F. C. Alcaraz, M. N. Barber, M. T. Batchelor, R. J. Baxter, and G. R. W. Quispel, J. Phys. A20, 6397 (1987).

[2] M. Gaudin, Phys. Rev. A4, 386 (1971).

[3] F. Woynarovich, Phys. Lett. 108A, 401 (1985).

[4] H. Schulz, J. Phys. C18, 581 (1985); H. Asakawa and M. Suzuki, preprint.

[5] T. Yamamoto, J. Phys. Soc. Jpn. 63, 1223 (1994); T. Yamamoto, N. Kawakami, and S. -K. Yang, J. Phys. A in press.

[6] I. Affleck and A. W. W. Ludwig, Nucl. Phys. B360, 641 (1991); B352, 849 (1991); Phys. Rev. Lett. 67, 161 (1991); Phys. Rev. B48, 7279 (1993); A. W. W. Ludwig and I. Affleck, Phys. Rev. Lett. 67 (1991) 3160; Nucl. Phys. B428, 545 (1994).

[7] S. Fujimoto, N. Kawakami, and S. -K. Yang, Phys. Rev. B50, 1046 (1994); J. Phys. Soc. Jpn. in press.

[8] J. L. Cardy, Nucl. Phys. B240, 514 (1984), ibid B324, (1989) 581; J. L. Cardy and D. C. Lewellen, Phys. Lett. B259, (1991) 274.

[9] B. Sutherland, Phys. Rev. Lett. 20, 98 (1968).

[10] P. Schlottmann, J. Phys. Condensed Matter 5, 5869 (1993).

[11] H. J. de Vega and F. Woynarovich, Nucl. Phys. B251, 439 (1985); F. Woynarovich, J. Phys. A22, 4243 (1989).

[12] In eq.(23), we omit the correction term of the zeroth order in $1 / L$ which corresponds to the non-universal surface energy of the boundaries, since it is irrelevant to the following discussion about the critical properties.

[13] H. W. Blöte, J. L. Cardy and M. P. Nightingale: Phys. Rev. Lett. 56 (1986) 742; I. 
Affleck, Phys. Rev. Lett. 56 (1986) 746.

[14] A.G. Izergin, V.E. Korepin and N.Y. Reshetikhin: J. Phys. A22 (1989) 2615.

[15] J.L. Cardy: Nucl. Phys. B 270[FS16] (1986) 186.

[16] N. Kawakami, Phys. Rev. B47, 2928 (1993).

[17] H. Frahm and A. Schadshneider, J. Phys. A26, 1463 (1993).

[18] T. Ogawa, A. Furusaki, and N. Nagaosa, Phys. Rev. Lett. 68, 3638 (1992).

[19] C. L. Kane, K. A. Matveev and L. I. Glazman, Phys. Rev. B49, 2253 (1994).

[20] N. V. Prokof'ev, Phys. Rev. B49, 2148 (1994).

[21] X. G. Wen, Int. J. Mod. Phys. B6, 1711 (1992).

[22] I. Affleck and A. W. W. Ludwig, J. Phys. A27, 5375 (1994). 